\title{
An Unmanned Aerial Traffic Management Solution for Cities Using an Air Parcel Model
}

\author{
Aislan Gomide Foina \\ Civil and Environmental Engineering \\ University of California, Berkeley, USA \\ Email: aislan@berkeley.edu
}

\author{
Clemens Krainer \\ Department of Computer Sciences \\ University of Salzburg, Austria \\ Email: ckrainer@cs.uni-salzburg.at
}

\author{
Raja Sengupta \\ Civil and Environmental Engineering \\ University of California, Berkeley, USA \\ Email: sengupta@ce.berkeley.edu
}

\begin{abstract}
The Unmanned Aerial System (UAS) market once belonged to professional companies only. Now many amateur pilots fly cheap, smartphone controlled models available in any electronic store. Due to altitude, speed, and weight, these flying objects can cause damage and injury. Hence, a way to manage them is necessary. This paper presents a UAS Traffic Management (UTM) system comprising three components: a UAS electronic identification plate with an embedded logger, a ground identification equipment, and a Traffic Routing System (TRS). This UTM system uses the air parcel model, which divides the low altitude airspace in a 3-D air parcel map. In this system, land parcel owners possess the airspace above their real estate and approve or disallow overflights. The adoption of the described UTM allows cities to control UASs, to detect non-compliant flights, and to identify pilots.
\end{abstract}

\section{INTRODUCTION}

Since 1981, remote controlled air models are allowed to fly in US airspace [1]. The conditions for flying are staying at a sufficient distance from populated and noise sensitive areas, flying at a maximum altitude of $120 \mathrm{~m}$ above ground, and keeping away from airports for more than $4.8 \mathrm{~km}$. With the evolution of technology, more electronics were embedded in the remote controlled air models, allowing them to make decisions and fly autonomously. As a consequence, Unmanned Air Vehicles (UAVs) were later renamed to Unmanned Aerial Systems (UASs). In 1990, the Federal Aviation Agency (FAA) first authorized the deployment of UASs in the National Air Space (NAS) for military or strategic use. However, back in 2013, Amazon showed their R\&D project pretending to use quadcopters to do express deliveries [2]. Several other companies, like DHL and Google, shared the same interest [3]. Due to the small size of these vehicles, the FAA is alleging lack of security or ways to manage it, refusing to authorize commercial applications of UASs. The actual UASs can be categorized by flight distance and altitude into three different groups:

- Long Haul UAS: Complete aerial vehicles for long haul inter-city or international flights. They are equipped with all the avionics necessary to fly in the controlled airspace and allowed to use the airports.

This work was supported in part by the Brazilian CNPq under Grant 232525/2013-7.
- Indoor UAS: Very small, cheap, and lightweight flying devices designed to fly indoors, with very limited battery life and slow speed. Maximum weight is less than one kilogram.

- Intra-city UAS: Quadcopters and fixed-wing planes designed to fly outdoor in low altitude uncontrolled class $\mathrm{G}$ airspace. Their flying time is around 30 minutes and their weight less than $3 \mathrm{~kg}$. They are also known as Small Unmanned Aircraft Vehicle (SUAV), used to reference man-portable UAVs [4].

There are many regulations and systems to manage long haul UAS, since they have all the sensors and equipment currently used by the civil aviation. The small indoor UAS do not have enough power to carry extra payload besides their own batteries and engines. Because of their small size and weight, they cannot cause much harm. Therefore, this paper presents a UAS Traffic Management (UTM) system for intracity UASs. In case UASs replace the traditional mail carriers in express delivery, we expect that many of the UASs will fly over the cities carrying payloads weighting less than one kilogram. The UTM general design is inspired in part by the actual motor vehicle traffic management systems used to regulate cars, trucks, roads, and highways. The UTM requires a license plate equivalent to be attached to UASs. The UTM also has a central system, the TRS, to manage UAS flights. The TRS applies the air parcel model to define permitted flight zones. With that, it calculates and schedules feasible flight tracks. Additionally, the UTM provides a ground device capable of identifying UASs, which allows inspection and surveillance.

\section{Air PARCEl Model}

The FAA divides the airspace in classes A to G. Space classes A, B, C, D, and E are controlled, that means an air traffic control (ATC) manages the airspace. All aircraft that want to fly through these airspaces need to be equipped with certain instruments and need to follow specific procedures. Class F is not used in the United States. Class G airspace is not controlled, i.e., there is no ATC to manage the airspace. Pilots are required to follow the visual flight rules (VFR) [5]. In the air parcel model the property owners also own the class $\mathrm{G}$ airspace above their plots of land up to a certain height. This airspace above a land parcel is called an air parcel. The air 


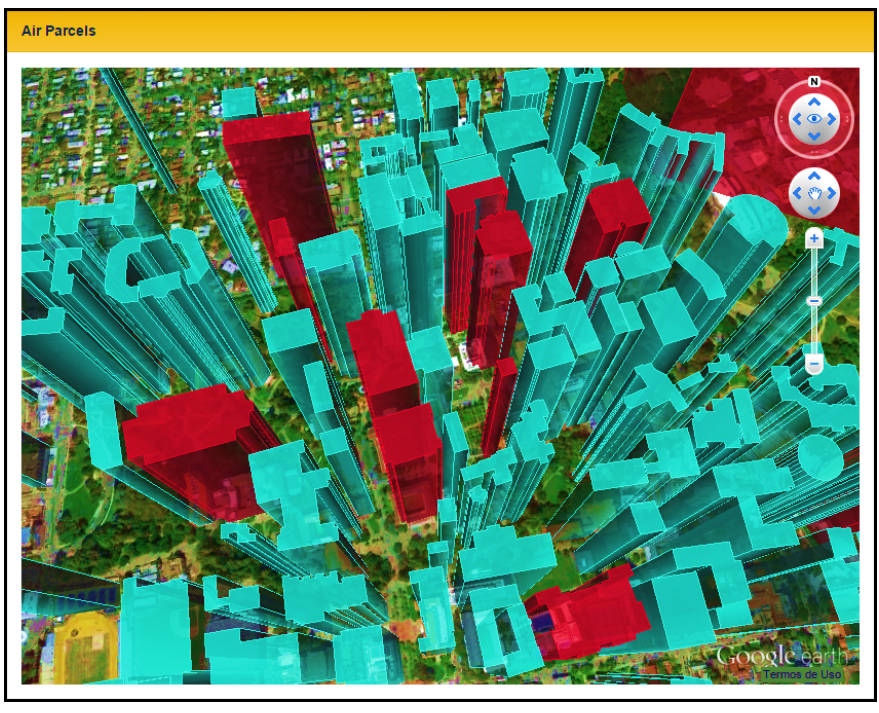

Fig. 1. A 3-D map of the UC Berkeley campus showing the air parcels of each building. Air parcels in red have the permission to fly through denied, while the cyan air parcels have no permission defined.

parcels over city roads rest with the city, the ones over county roads with the county, and the ones over other properties with the respective landowners. The idea is the distribution of UTM responsibility to be confluent with the federated system of government and pattern of property ownership. In the TRS each air parcel data set has annotations specifying what kind of UASs may enter the particular parcel for which purposes and times. An air parcel owner would publish her UTM policy using these annotations. A celebrity might publish a deny policy like "no UAS Access" to protect her privacy. A city enabling UAS package delivery over its roads would provide more real UTM with Command \& Control, Detect \& Avoid, or Ground Ops. Just as road maps came to be built in data structures enabling real-time routing and navigation apps for drivers, air parcel maps also have to be built to promote realtime apps for UAS pilots. Figure 1 shows an example of the 3-D air parcel visualization and air parcel permissions.

\section{AIR PARCEL UTM}

Using the air parcel model and some rules of the existing ground vehicle traffic management systems, we created the Air Parcel UTM presented it in this paper. Since the UTM concept was introduced recently by NASA [6], the bibliography available focuses more on systems to manage several UASs at the same time, or to avoid collisions in UAS fleets. In the collision avoidance research field, one paper considers the aircraft as agents and the airspace as cells. It uses graph theory to calculate trajectories and to estimate collisions. The authors use agent prioritization to resolve collisions [7]. Other papers describe the approximation of aircraft dynamics using only linear constraints. This allows applying a mixed-integer linear program (MILP) approach to create routes without collisions [8], [9]. These papers need real-time information from the vehicles in order to avoid collisions and they do not solve identification issues in case of non-conform situations. In the
UTM design described in this paper, we believe a UAS must handle the collision avoidance autonomously using sensors to detect obstacles and change the route as well as the altitude by itself. This works in the same way as autonomous cars in research projects and the actual civil aviation.

Focusing on systems to manage several UAS, some authors analyze the vehicle-to-operator ratio and try to increase this number managing by exception or managing by consent approaches [10]. Other authors have defined control loops that focus in improving this ratio. They point out the reduced awareness the pilot will have when controlling several UAS at the same time [11]. Considering that thousands of UAS will be flying over the cities, it is impractical to allocate one operator for each group of aircraft.

More directly related to a complete UTM function, some papers describe the challenges of using UASs. They describe the technical challenges of developing a UAS based system for a smart cities context [12] and NAS integration [13], [14]. A project called The Smart Skies Project proposes three systems working together: (1) a Mobile Aircraft Tracking System (MATS), i.e., a ground station to track UASs; (2) a vehicle onboard Sense-and-Act (SA) system, which allows the UAS to detect obstacles; and (3) a central Automated Separation Management System (ASMS), to receive the UAS data as well as to correct UAS routes [15]. This system also relies on real-time data transmitted by the UASs in order to feed a database and make decisions. Another project has created a flight planning system that receives information from a ground sensor network, mostly meteorological data, as well as positioning and collision detection information from the UAS [16]. None of these systems provide tools for UAS identification to other parties, like civilians. These systems also rely on working communication links between UASs and the central system to create a closed control loop. Hence, communication loss causes serious problems.

Finally, some patents provide identification of UAS using RF and color codes. One patent using RFID describes a box embedded in the drone that allows taking control by request from a ground station [17]. Another patent describes the communication between a traffic control system and a box embedded in the UAS [18]. There is one patent using light signals to identify UAVs. It describes the use of an illuminated encoded signal to detect friend-or-foe aircraft [19]. Both approaches only allow the identification by special devices. In this paper, the identification mechanism is based on visible LED color patterns, in order to allow any civilian to look at the sequence and trace-back the UAS when necessary. This is one of the principles adopted in our UTM.

The Air Parcel UTM architecture contains three components: the electronic license plate, the Traffic Routing System (TRS), and a ground identification device.

\section{A. Electronic License Plate}

A traffic management system monitors the traffic and notifies the responsible enforcement units in case of any noncompliance with existing regulations. Therefore, the first step 
to identify the responsible party is to identify the vehicle. The ground traffic management system requires all vehicles to have a license plate for driving through public roads. This plate allows the police and other drivers to identify a vehicle. Similarly, in order to manage UASs, the UTM needs something equivalent to a license plate.

Instead of stamping letters and numbers in aerial vehicles, we propose using a LED array that blinks in a unique color sequence for each UAS. The advantage of this identification mechanism is the possibility for any person to visually identify any UAS from long distances without the use of any special equipment. The UTM defines the license plate color sequence. The number of colors available and the number of blinks define the quantity of codes possible.

Additionally to the LED array, the electronic license plate has a position logger and a small UHF transponder. The logger reads the GPS position from the UAS GPS receiver or from its own GPS receiver. It stores all the flight data of each trip made by the UAS. This information is the evidence of the real UAS flight path, in case somebody claims that this UAS was seen in a different place.

The transponder broadcasts the same code as blinked by the LEDs via UHF frequency. Moreover, the transponder transmits the current UAS position, allowing the implementation of a collision avoidance system. The communication between transponder and ground receiver is unidirectional, where only the transponder transmits. In case of a vehicle crash, the transponder will help to find the wrecked UAS. Furthermore, the position logger will have all the information about the flight, similar to the black box installed on civil airplanes.

\section{B. Traffic Routing System}

The brain our UTM is the TRS. It receives all the routing requests from the pilots to calculate an efficient and collision free trajectory. Overflight of property might be restricted in time and might cost money. Hence, the TRS calculates several route options, showing the total distance to fly, the total cost to be paid, and the available schedules.

A pilot uploads a planned UAS trajectory to the TRS as a list of waypoints. The TRS assumes a straight flying path between successive waypoints. Depending on occupation, permissions, and time constraints of each crossed air parcel, the TRS calculates a set of possible routes. The TRS will try approve the pilot's planned route without modification, but if there is any restricted air parcel in the path, the TRS will change the route. The TRS might add waypoints to a route to avoid crossing no-fly zones, it would modify a route to avoid UAS congestion, and it could suggest to reschedule the flight. For instance, if the air parcels for the shortest route are too busy in the departure time the pilot requested, the TRS will calculate a longer route for that specific time considering other air parcels. However, if in a few minutes later these air parcels are available, the TRS will also generate an optional route considering these air parcels with a later departure time. The TRS presents the estimated feasible routes to the pilot, who selects his desired route depending on trajectory, speed,

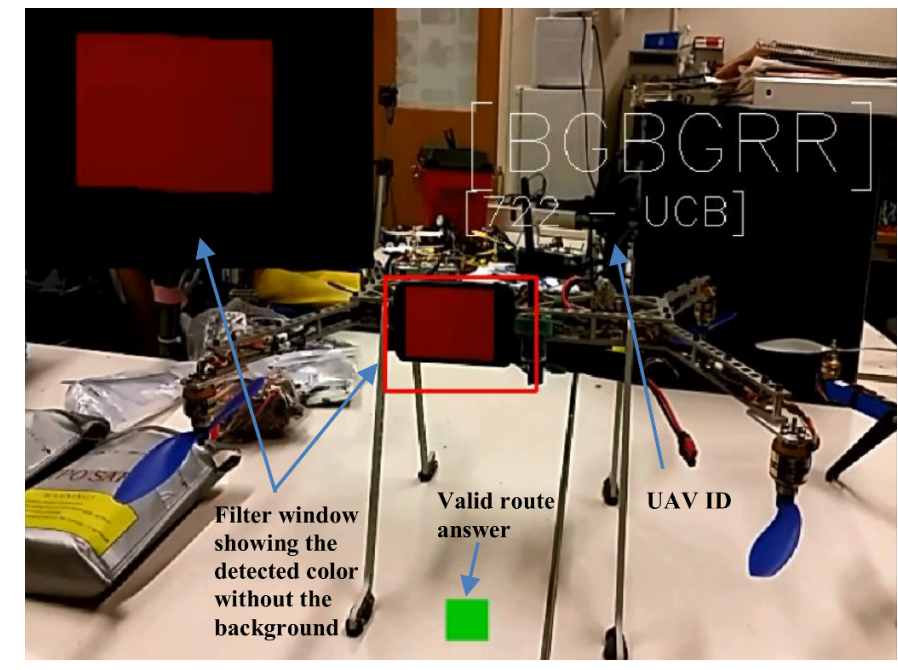

Fig. 2. The user interface of the ground identification device, showing the "valid" green box in case of correct detection of a UAS on its selected route.

and costs. After that, the pilot downloads the chosen trajectory as a GPS waypoint list to program the UAS.

Our TRS does not require continuous communication with all flying UASs, but after each flight the TRS must receive the flight path captured by a UAS license plate in order to proof the correct execution of the route.

\section{Ground Identification Device}

Nowadays the police department maintains law enforcement agents supervising the roads and highways in order to detect drivers disobeying traffic laws. Devices like the radar allow police officers velocimetry. Moreover, the license plate allows identifying the vehicle owner and accessing the complete vehicle history. A control system similar to this for airspace management is also necessary to allow UAS surveillance and regulation.

Since the UAS identification relies on visual information, we developed a special camera device with image processing algorithms to capture the license plate color sequence. This sequence and the position the UAS was detected at are submitted to the TRS for validation. The TRS compares the received information with the routing position and time data for that UAS. Hence, if the inspection officer wants to check if one specific vehicle is following the correct path, he just points the identification device to the UAS. The device automatically handles the identification and crosschecks with the TRS. After the TRS replies, the device shows on the screen if that UAS position and timestamp is according to its route. In positive case, the device screen shows a green square as a "valid" feedback, as shown in Figure 2. Otherwise, the user will see an red square as "error" message.

\section{Process Workflow}

The combination of the described UTM components allows the complete control of UAS flights without the necessity of continuous communication connections to the central management system. To comply with the UTM, the pilots must follow 


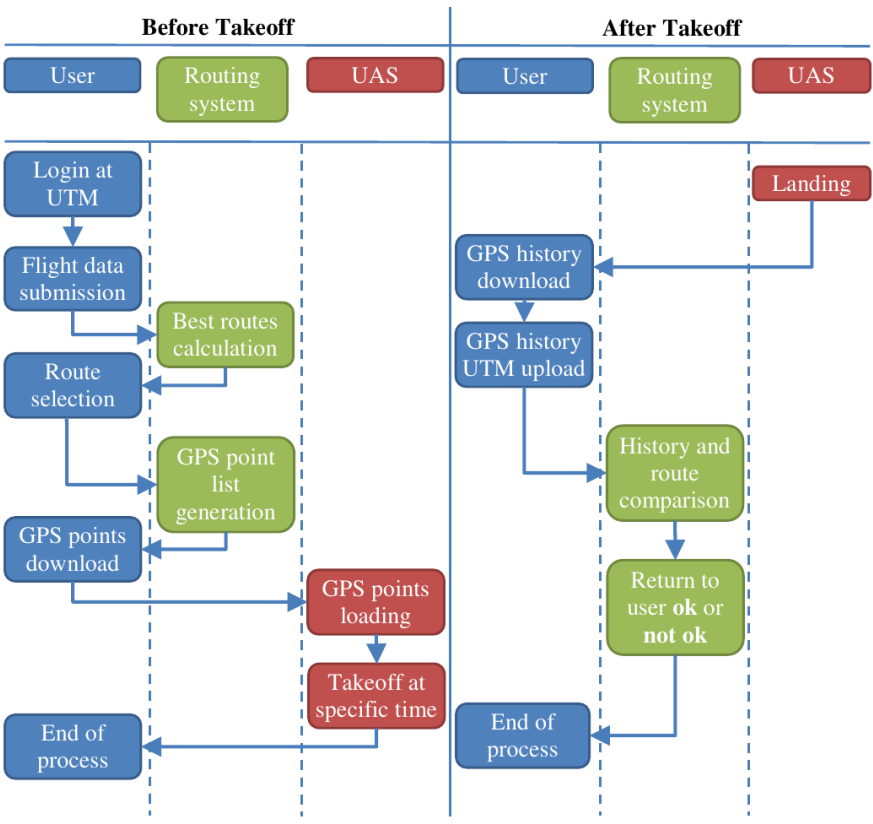

Fig. 3. Process flow from request data submission to real flight data upload

a sequence of procedures before, during, and after the flight. Figure 3 presents this data flow.

Before takeoff, the pilot submits the projected route to the TRS as a list of waypoints and the intended take off time. The TRS receives the projected route and checks air parcel permissions and occupancy in order to calculate feasible routes and departure time options per route. The pilot selects one of the proposed routes and downloads it in GPX format [20]. He programs his UAS to follow these points and to departure at the assigned departure time.

After loading all the information into the UAS, the pilot launches the UAS in time to execute the flight plan. The UAS license plate position logger captures the entire executed flight path. After landing, the pilot has to download the GPS log from the position logger and upload it to the TRS for future auditing. In case of available Internet access provided by Wi-Fi access points or cellular networks the license plate hardware automatically uploads the GPS log. Figure 4 shows a postflight path visualization and the list of air parcels crossed by the UAV. If, at any time, somebody sees this UAS while flying and wants to check if it is following the scheduled route, it is just a matter of submitting the time and position where the UAS has been seen to the UTM. The UTM response contains the state, the pilot, and the route.

\section{Tests Results}

Our first license plate prototype used a Raspberry PI, which was connected to several RGB LEDs and received GPS coordinates via the Mavlink protocol from a PX4 autopilot. However, to create a standalone solution without the necessity of integrating with autopilots, we applied an Android mobile phone as a second version of the license plate prototype. Instead of the LEDs, the screen shows the color sequence, and a small Android app reads the current position from the phone's

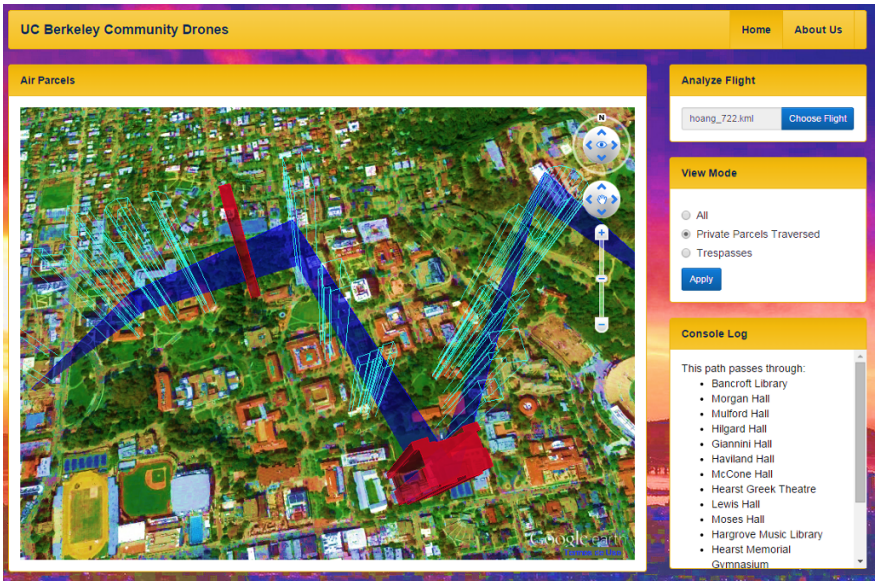

Fig. 4. In blue is the 3-D visualization of a UAS flight, showing each air parcel crossed. In the bottom right window is listed all the air parcels crossed, and the air parcel trespasses are highlighted in red in the map.

GPS receiver and stores the data in files. Depending on WiFi or $3 \mathrm{G}$ network availability, the Android app automatically uploads the trajectory data to the UTM.

For the tests, we defined the blink pattern to use only six different colors, red, green, blue, cyan, yellow and pink. The sequence consists of six blinks, creating 46,656 possible combinations. The license box shows each color for $500 \mathrm{~ms}$. Between colors is a $200 \mathrm{~ms}$ pause to separate the blinks. Since the sequence is blinked in a loop, there is a onesecond pause at the sequence end in order to visually define when the sequence begins and ends. The maximum total time necessary to capture the complete blink sequence is $5 \mathrm{~s}$. Changing parameters like the number of blinks, colors and blinking duration, supports more or less combinations and different total blinking duration. We used a sequence of six color flashes to allow persons looking at a UAS to be able to see and memorize the complete sequence. We also used the smartphone Bluetooth 4.0 radio to simulate the license plate transponder, where the Bluetooth device name contains the license plate numeric identification and the last five digits of the decimal GPS coordinates.

In order to increase the visibility from ground, the color LEDs and the mobile phone were installed on the bottom side of the drone, facing to the ground, as depicted in Figure 5. Using a 4" screen phone installed on the drone, we could archive a maximum visual distance of $87 \mathrm{~m}$ during a sunny the day and $384 \mathrm{~m}$ at night.

The ground identification device prototype is another Android cell phone that has a special lens attached to it. The cell phone camera captures the image, and a 12x external lens increases the detection range. Figure 6 shows the device used for the tests. We developed an Android app that uses the OpenCV [21] library to detect the colors. By applying this lens, the detection range increases around 30\%, to $104 \mathrm{~m}$ during the day and $516 \mathrm{~m}$ at night. Table I presents all the measured distances.

As expected, we could not use blue in the identification color sequence. The background sky is mostly blue during 


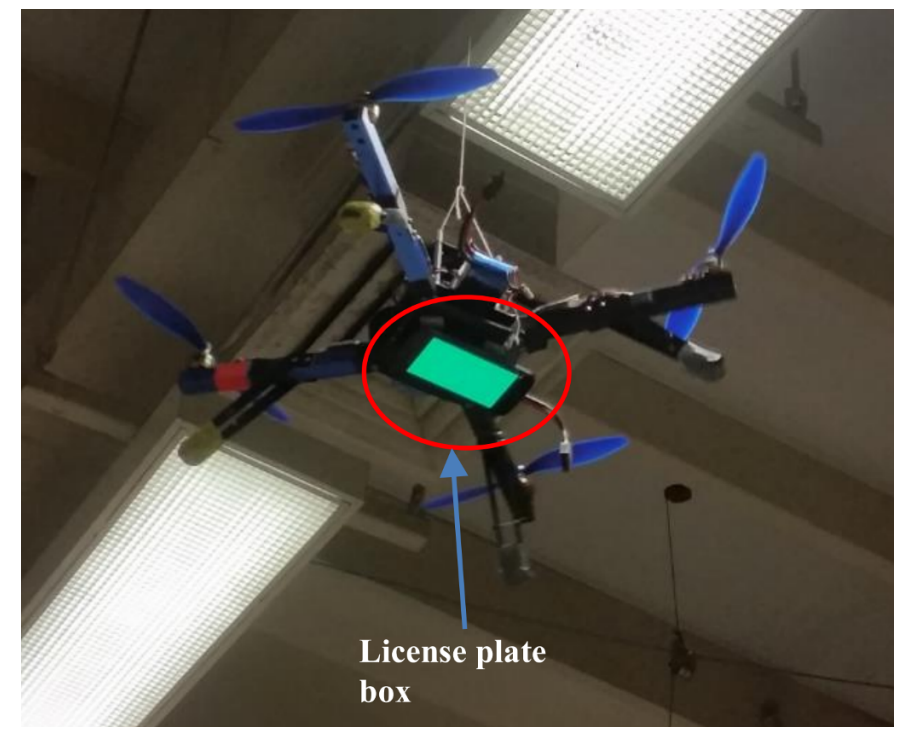

Fig. 5. License plate box mounted on the underside of a quadcopters.

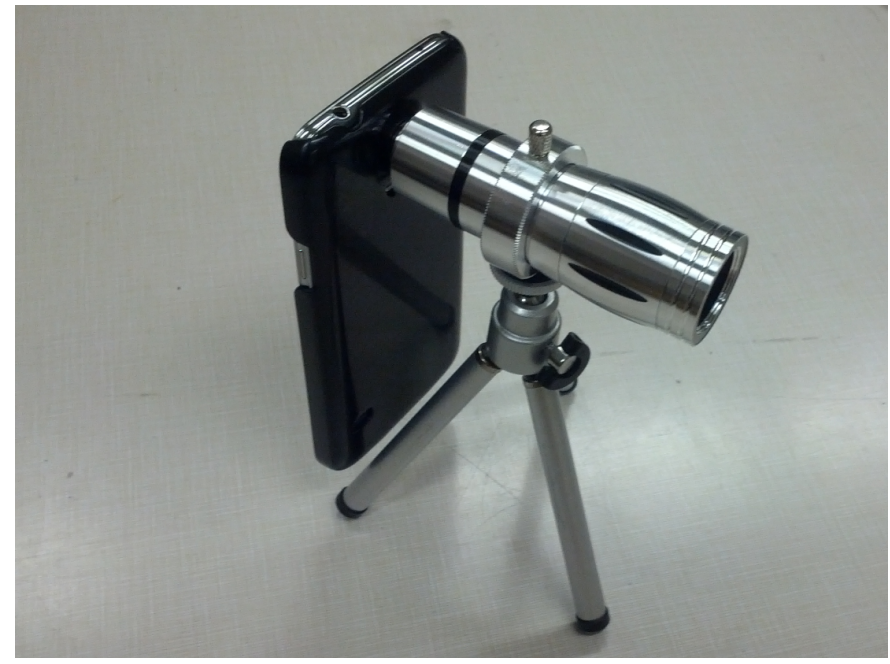

Fig. 6. The prototype of the ground identification device.

the day and the app was not able to distinguish between the license plate and the sky. In addition, when the vehicle is exactly between the ground device and the sun, the algorithm has problems to identify the UAS blinks. The sun is much brighter than the mobile screen, causing difficulties to identify the blinks and the colors. For this reason, we moved back the license plate development to use LEDs instead of a LCD screen. Comparing to ground vehicles break lights, LEDs are visible by more than $300 \mathrm{~m}$ on roads, even against the sun.

\section{Full Scale UTM Adoption}

In order to realize a large-scale adoption of the UTM presented here, we defined some premises and some tasks that have to be done.

1) Landowners possess their air parcel and should grant the permission for crossing their property.

The city council defines the default rule as allowed or denied, but landowners can override it at any time. They
TABLE I

LiCEnSE Plate Color Identification RanGe

\begin{tabular}{|c|c|c|c|}
\hline Light Emitter & Environment Light & Light Receiver & Range \\
\hline \multirow{6}{*}{$\begin{array}{l}\text { Smartphone } \\
\text { Screen }\end{array}$} & \multirow{3}{*}{ Day } & Camera & $15 \mathrm{~m}$ \\
\hline & & Camera $12 x$ & $104 \mathrm{~m}$ \\
\hline & & Eyes & $87 \mathrm{~m}$ \\
\hline & \multirow{3}{*}{ Night } & Camera & $42 \mathrm{~m}$ \\
\hline & & Camera $12 \mathrm{x}$ & $516 \mathrm{~m}$ \\
\hline & & Eyes & $384 \mathrm{~m}$ \\
\hline \multirow{6}{*}{$\begin{array}{l}\text { Ultrabright } \\
\text { RGB LEDs }\end{array}$} & \multirow{3}{*}{ Day } & Camera & $24 \mathrm{~m}$ \\
\hline & & Camera $12 \mathrm{x}$ & $158 \mathrm{~m}$ \\
\hline & & Eyes & $101 \mathrm{~m}$ \\
\hline & \multirow{3}{*}{ Night } & Camera & $55 \mathrm{~m}$ \\
\hline & & Camera $12 \mathrm{x}$ & $683 \mathrm{~m}$ \\
\hline & & Eyes & $461 \mathrm{~m}$ \\
\hline
\end{tabular}

can even charge pilots for crossing their air parcel and, depending on time, vary price as well as transit permits. For example, at night and on weekends a landowner may deny crossing his air parcel, but from 9 AM to 5 PM on working days, flights are allowed and will cost some amount of money per minute of overflight.

2) City councils are responsible for the uncontrolled low altitude class $G$ airspace inside their territory.

The FAA needs to waive the regulation of the class $G$ low-altitude airspace over cities. Defining and maintaining airspaces where UAS may fly must belong to a city council. For example, busy avenues and highways are restricted for UAS to fly, public schools and university areas as well during class hours. In this model, the FAA will control only the high altitude class $G$ and the controlled airspace. This premise will help the cities to benefit from the UAS market. They could charge annually fees for each drone registered at the UTM, similarly as the fees charged for car license plates. This charge could be used to finance the UTM operation. For the pilot, the payment of the UTM fee gives him permission to fly over the city. Licensed pilots can send UASs to fly over any uninhabited piece of land inside the city limits and any allowed air parcel. The pilots also will have access to use the TRS and will receive a license plate code for each registered UAS. In addition, the citizens have a local authority for complaining about irregular flights, they will not need to write a federal complain to FAA in case of a UAS flying in a "NOUAS" area.

3) All flying UAS must have the license plate.

The first obligation of a pilot is to maintain the UAS license plate installed and working before and when the UAS flies. The law enforcement department responsible for the UTM operation must be capable of identifying a flying UAS to check its permission in the TRS. Any UAS without the plate can suffer the law enforcement defined by the city. The consequences of violations could be monetary penalties, as well as pursuit, capture, and destruction of a UAS. 
4) All pilots must submit their flight plans to the TRS. The TRS stores all UAS flight data and allows reports on air parcel utilization. Any UAS flying over the city without its route registered in the TRS may collide with other UASs. Hence, all pilots must submit their flight plans and follow the trajectories calculated by the TRS.

5) All UAS must fly only the chosen route at the scheduled time.

After a pilot uploads the flight plan and chooses a specific calculated route at a specific time, he must follow this route. In case of modifications in the flight plan, the pilot must submit the new plan to the TRS to get a new route and schedule. If the pilot fails to follow this procedure, the UAS law enforcement unit can penalize the pilot in case of detection by the ground identification device.

\section{CONCLUSION AND FUtURE WORKS}

The UTM using the air parcel model is a viable solution for the UAS regulation issue. The adoption by the city is not technologically challenging, it needs a web based, highly available TRS, which could be conveniently based on available cloud services. Pilots just attach the license plate hardware on the bottom side of their UASs. After that, it is just a matter of populating the TRS database with the landowner's permission settings, UAS registrations and finally, start to receive requests and generate the routes.

The air parcel model is suitable for cities to solve the UAS regulation problem for short outdoor flights. Its concept is natural for the users because it follows the same premises of the land rule. It gives the airspace control to the local city council and allows the property owners to exploit their airspace.

For visually identification of UAS, RGB LEDs show better test results than smartphone screens. Discrete LEDs have much more luminous intensity than the LEDs used in smartphone screen backlights. Therefore, one future improvement will be to add several high intensity RGB LEDs on the bottom side of UAS frames to increase the visual range to at least $200 \mathrm{~m}$ during daytime. The LEDs will consume more power than the current solution, but compared to the power consumed by the DC engines, the UAS total flight time will not shorten perceivably.

The ground identification device needs a more advanced image-processing algorithm. In the actual stage, the user must follow the UAS with the camera and keep it in the middle of the image. Adding color reference LEDs in the ends of the frame should allow recognizing the shape of the UAS and detecting the color sequence without centralizing the UAS in the camera image. Advanced video processing techniques together with other sensor input could be used to even guess the flight direction [22]. These two features permit the construction of a ground turret that automatically scans the sky, detects UAS, and reports them to the TRS. Then, the TRS checks flight plans without human interaction and notifies the responsible parties in case of punishable acts.
Finally, the TRS needs some easy way to import the 2D land map and to generate the air parcels. Integration with GPS map databases should provide the automatic air parcel generation for houses, buildings, and roads.

\section{REFERENCES}

[1] Federal Aviation Administration, "Model Aircraft Operating Standards," Advisory Circular 91-57, 1981.

[2] Amazon. (2015) Amazon Prime Air. [Online]. Available: http: //www.amazon.com/b?node $=8037720011$

[3] A. Barr and G. Bensinger, "Google Is Testing Delivery Drone System," Wall Street Journal, Aug. 29, 2014.

[4] UAS America Fund. (2014) Unmanned Aerial Systems - A Path Forward. [Online]. Available: http://www.uasamericafund.com/assets/ uasfund_mua-proposed-part107_dec2014.pdf

[5] Pilots Handbook of Aeronautical Knowledge. Federal Aviation Administration, 2008, vol. FAA-H-8083-25A, Chapter 14.

[6] P. H. Kopardekar, "Unmanned Aerial System (UAS) Traffic Management (UTM): Enabling Low-Altitude Airspace and UAS Operations," NASA Ames Research Center, Tech. Rep., Apr. 1, 2014.

[7] S. Resmerita, M. Heymann, and G. Meyer, "A framework for conflict resolution in air traffic management," in Proc. 42nd IEEE Conference on Decision and Control, vol. 2, Dec. 2003, pp. 2035-2040 Vol.2.

[8] A. Richards and J. How, "Aircraft trajectory planning with collision avoidance using mixed integer linear programming," in Proc. American Control Conference, vol. 3, May 2002, pp. 1936-1941 vol.3.

[9] A. Alonso-Ayuso, L. Escudero, and F. Martin-Campo, "Collision Avoidance in Air Traffic Management: A Mixed-Integer Linear Optimization Approach," IEEE Transactions on Intelligent Transportation Systems, vol. 12, no. 1, pp. 47-57, Mar. 2011.

[10] D. Liu, R. Wasson, and D. Vincenzi, "Effects of System Automation Management Strategies and Multi-mission Operator-to-vehicle Ratio on Operator Performance in UAV Systems," Journal of Intelligent and Robotic Systems, vol. 54, no. 5, pp. 795-810, 2009.

[11] M. Cummings, S. Bruni, S. Mercier, and P. Mitchell, "Automation architecture for single operator, multiple uav command and control," Massachusetts Inst. of Tech. Cambridge, Tech. Rep., 2007.

[12] F. Mohammed, A. Idries, N. Mohamed, J. Al-Jaroodi, and I. Jawhar, "UAVs for smart cities: Opportunities and challenges," in International Conference on Unmanned Aircraft Systems (ICUAS), May 2014, pp. 267-273.

[13] M. DeGarmo and G. Nelson, "Prospective Unmanned Aerial Vehicle Operations in the Future National Airspace System," AIAA 4th Aviation Technology, Integration, and Operations (ATIO) Forum, vol. AIAA6243, Sep. 2004.

[14] A. Simpson and J. Stoker, "Safety Challenges in Flying UAVs (Unmanned Aerial Vehicles) in Non Segregated Airspace," in The 1st Institution of Engineering and Technology International Conference on System Safety, Jun. 2006, pp. 81-88.

[15] R. Clothier, R. Walker, R. Baumeister, M. Brunig, J. Roberts, A. Duggan, and M. Wilson, "The Smart Skies project," Aerospace and Electronic Systems Magazine, IEEE, vol. 26, no. 6, pp. 14-23, Jun. 2011.

[16] M. Aubert, S. Uzumcu, A. Hutchins, and M. Cummings, "Toward the Development of a Low-Altitude Air Traffic Control Paradigm for Networks of Small, Autonomous Unmanned Aerial Vehicles," in AIAA SciTech Conference, Kissimmee, FL, Jan. 2015.

[17] J. Matos, "Systems and methods for detecting and managing the unauthorized use of an unmanned aircraft," Jan. 26, 2012, US Patent App. 13/195,268 Available: https://www.google.tl/patents/US20120022719.

[18] D. Limbaugh, D. Barnhard, and T. Rychener, "Unmanned aerial system position reporting system," Feb. 26, 2013, US Patent 8,386,175 Available: https://www.google.tl/patents/US8386175.

[19] I. Kymissis, "Friend or foe detection," Oct. 21, 2010, US Patent App. 12/682,216 Available: https://www.google.t1/patents/US20100266126.

[20] D. Foster, "GPX: the GPS Exchange Format," Available: http://www. topografix.com/gpx.asp, [Accessed: Jan. 23, 2015].

[21] Open Source Computer Vision Library. Available: http://opencv.org. [Accessed: Jan. 23, 2015].

[22] M. Jackson, Y. Zhao, and R. Slattery, "Sensitivity of Trajectory Prediction in Air Traffic Management," Journal of Guidance, Control, and Dynamics, vol. 22, no. 2, pp. 219-228, 1999. 